\title{
Development of PLGA-lipid nanoparticles with covalently conjugated indocyanine green as a versatile nanoplatform for tumor-targeted imaging and drug delivery
}

\author{
This article was published in the following Dove Press journal: \\ International Journal of Nanomedicine \\ 4 November 2016 \\ Number of times this article has been viewed
}

Yu Xin

Tie Liu

Chenlong Yang

Department of Neurosurgery, Beijing Tiantan Hospital, Capital Medical University, Beijing, People's Republic of China
Correspondence: Yu Xin Department of Neurosurgery, Beijing Tiantan Hospital, Capital Medical University, No 6, Tiantan XiLi, Beijing 100050, People's Republic of China

Tel +86139 II 707098

Fax +8601067098431

Email yuxin_new@163.com
Abstract: We have prepared novel poly(D,L-lactide-co-glycolide) (PLGA) lipid nanoparticles (PNPs) that covalently conjugate folic acid (FA) and indocyanine green (ICG), in addition to encapsulating resveratrol (RSV) (FA-RSV/ICG-PLGA-lipid NPs, abbreviated as FA-RIPNPs); these nanoparticles have been developed for simultaneous targeted delivery of anticancer drug and fluorescence imaging. The FA-RIPNPs, with an average particle size of $92.8 \pm 2.1 \mathrm{~nm}$, were prepared by a facile self-assembly-and-nanoprecipitation method, and they showed excellent stability and biocompatibility characteristics. The FA-RIPNPs exhibited an RSV encapsulation efficiency of approximately $65.6 \% \pm 4.7 \%$ and a maximum release ratio of $78.2 \% \pm 4.1 \%$ at $\mathrm{pH} 5.0$ and $37^{\circ} \mathrm{C}$. Confocal fluorescence images showed that FA-RIPNPs may facilitate a high cellular uptake via FA receptor-mediated endocytosis. Furthermore, FA-RIPNPs (containing $50 \mu \mathrm{g} / \mathrm{mL} \mathrm{RSV}$ ) induced a $81.4 \% \pm 2.1 \%$ U87 cell inhibition rate via apoptosis, a value that proved to be higher than what has been shown for free RSV $(53.1 \% \pm 1.1 \%$, equivalent RSV concentration). With a formulated polyethylene glycol (PEG) shell around the PLGA core, FA-RIPNPs prolonged the blood circulation of both free RSV and ICG, which approximately increased 6.96- and 39.4-fold $\left(t_{1 / 2}\right)$, respectively. Regarding FA-RIPNP use as a near-infrared probe, in vivo fluorescence images indicated a highly efficient accumulation of FA-RIPNPs in the tumor tissue, which proved to be approximately 2.8- and 12.6-fold higher than the RIPNPs and free ICG, respectively. Intravenous injection of FA-RIPNPs into U87 tumor-bearing mice demonstrated the best tumor inhibition effect for all tested drugs, including free RSV and RIPNPs, with no relapse, showing high biocompatibility and with no significant systemic in vivo toxicity over the course of the treatment ( 1 month). The results obtained demonstrate the versatility of the NPs, featuring stable fluorescence and tumor-targeting characteristics, with promising future applications in cancer therapy.

Keywords: indocyanine green, resveratrol, near-infrared nanoprobe, tumor targeting, fluorescence imaging, drug delivery

\section{Introduction}

Brain glioma is a primary and commonly diagnosed brain tumor species with a high mortality rate within 1-2 years following diagnosis. ${ }^{1}$ A critical feature of glioma is that the tumor does not feature a defined border, which renders complete surgical removal of the tumor very difficult. The standard treatment for glioma is surgical excision combined with adjunctive radiotherapy. However, serious side effects and poor survival rates are associated with the therapy of this cancer type. ${ }^{2}$ A precise diagnosis for 
surgical excision and targeted chemotherapy with minimal side effects are critical, albeit unmet, scientific goals and an ideal strategy in the treatment of glioma. Near-infrared (NIR) fluorescence imaging, combined with an actively targeting probe with high sensitivity, imaging speed and tumortargeted imaging effect, has been developed as a promising approach for the precise and intraoperative identification of tumor margins. ${ }^{3-7}$ Recently, various researchers have reported that NIR fluorescence imaging using various NIR probes shows promising results for the identification of tumor tissues in both preclinical and pilot clinical studies. ${ }^{3,6}$

Indocyanine green (ICG), an amphiphilic tricarbocyanine dye, is a US Food and Drug Administration-approved NIR clinical fluorescence imaging probe. ${ }^{8,9}$ Due to low toxicity and excellent fluorescence characteristics, ICG - a probe that can absorb and emit in the NIR region (650-900 nm) and penetrates into deeper tissues with low tissue absorption and autofluorescence - has been widely applied in disease diagnosis, including the test of cardiac output, hepatic function and ophthalmic perfusion..$^{9-12}$ Nevertheless, effective and prolonged tumor imaging applications of ICG are restricted by a tendency of the compound to aggregate and degrade in aqueous solutions with concentration dependence, poor photostability, lack of target specificity and quick body clearance $\left(t_{1 / 2}=2-4 \mathrm{~min}\right) .{ }^{13-16}$ To overcome these drawbacks, various nanoparticle formulations of ICG, such as encapsulation approaches using polymer and liposomes, as well as physical adsorption with 2-dimensional materials, including graphene and derivatives, transition metal dichalcogenides and so on, have been reported. ${ }^{16-19}$ However, noncovalent interactions tend to be weak in nature, often resulting in premature release of the compound from the carriers, which may influence the imaging applicability of the nanoprobes. ${ }^{20}$ Therefore, covalent conjugation of ICG with nanomaterials may feature a more rational strategy for ICG delivery.

Resveratrol (RSV) is a type of natural antioxidant and nonflavonoid polyphenol derived from various dietary sources, including grapes, soybeans and so on. ${ }^{21}$ To date, RSV has been widely used in preclinical applications, such as inhibition of platelet aggregation, vasodilation, blood viscosity reduction as well as prevention of cancer occurrence and development. ${ }^{22-24}$ Recently, some research groups have found that RSV exhibits a great anticancer effect in various cancer types, such as colorectal cancer, breast cancer, glioma and so on. ${ }^{22,25,26}$ However, RSV still possesses some features, which limit its extensive use, such as poor solubility, low bioavailability and compound instability. For instance, the change from trans-isomer to cis-isomer can reduce the biological activity of RSV significantly. ${ }^{27-29}$ Therefore, various new strategies, including encapsulation of RSV into lipid or polymer-based nanoparticles, delivery using carbon nanomaterials and so on, have been developed in an effort to protect the structural integrity of the bioactive molecule. ${ }^{29-31}$ And these carriers showed high RSV loading capacity, enabling their use in biomedical applications. ${ }^{32-34}$

In this study, a formulation based on poly(D,L-lactideco-glycolide) (PLGA) lipid nanoparticles (PNPs) that covalently conjugate ICG and folic acid (FA) - a tumor targeting molecule, ${ }^{35,36}$ in addition to encapsulating RSV, has been prepared (FA-RSV/ICG-PLGA-lipid NPs, abbreviated as FA-RIPNPs) as a targeted NIR probe and RSV carrier for U87 glioma theranostic applications by using a facile self-assembly method (Scheme 1). It has been reported that the surfaces of U87 cells show overexpression of the folate receptor (FR), which has been used to develop FA-mediated targeting chemotherapy. ${ }^{2}$ The prepared nanoparticles (FARIPNPs) demonstrate great biocompatibility and stability, in addition to prolonging the blood circulation of RSV and ICG. In addition, FA-RIPNPs were used as NIR probes to monitor the cell internalization, in vivo biodistribution and tumor accumulation of RSV. At the same time, the targeted anticancer efficacy of FA-RIPNPs was evaluated in vitro and in vivo. The results obtained indicate that FA-RIPNPs may represent a versatile nanoplatform useful as a potential tumor theranostic agent that simultaneously enables precise diagnosis for clinical surgery and further targeted chemotherapy applications.

\section{Materials and methods Materials}

ICG, RSV ( $\geq 99 \%$ ) and PLGA (molecular weight: 5,00015,000 Da; lactide: glycolide ratio, 50:50) were purchased from Sigma Aldrich (St Louis, MO, USA). 4',6Diamidino-2-phenylindole (DAPI) was obtained from Aladdin (Shanghai, China). Soybean lecithin consisting of 90\%-95\% phosphatidylcholine, 1,2-distearoyl-sn-glycero-3 -phosphoethanolamine-N-[folate (polyethylene glycol)-2000] (DSPE-PEG ${ }_{2 \mathrm{k}}-\mathrm{FA}$ ) and 1,2-distearoyl-sn-glycero-3-phosphoethanolamine-N-[carboxy (polyethylene glycol)-2000] (DSPE-PEG ${ }_{2 \mathrm{k}}-\mathrm{COOH}$ ) were obtained from Avanti (Alabaster, AL, USA). N-hydroxysuccinimide (NHS) and 1-ethyl-3-(3dimethylaminopropyl) carbodiimide (EDC) were obtained from Sigma Aldrich. All cell culture reagents, including trypsin-ethylenediaminetetraacetic acid solution, phosphatebuffered saline (PBS), fetal bovine serum (FBS), penicillinstreptomycin solution and minimum essential medium 


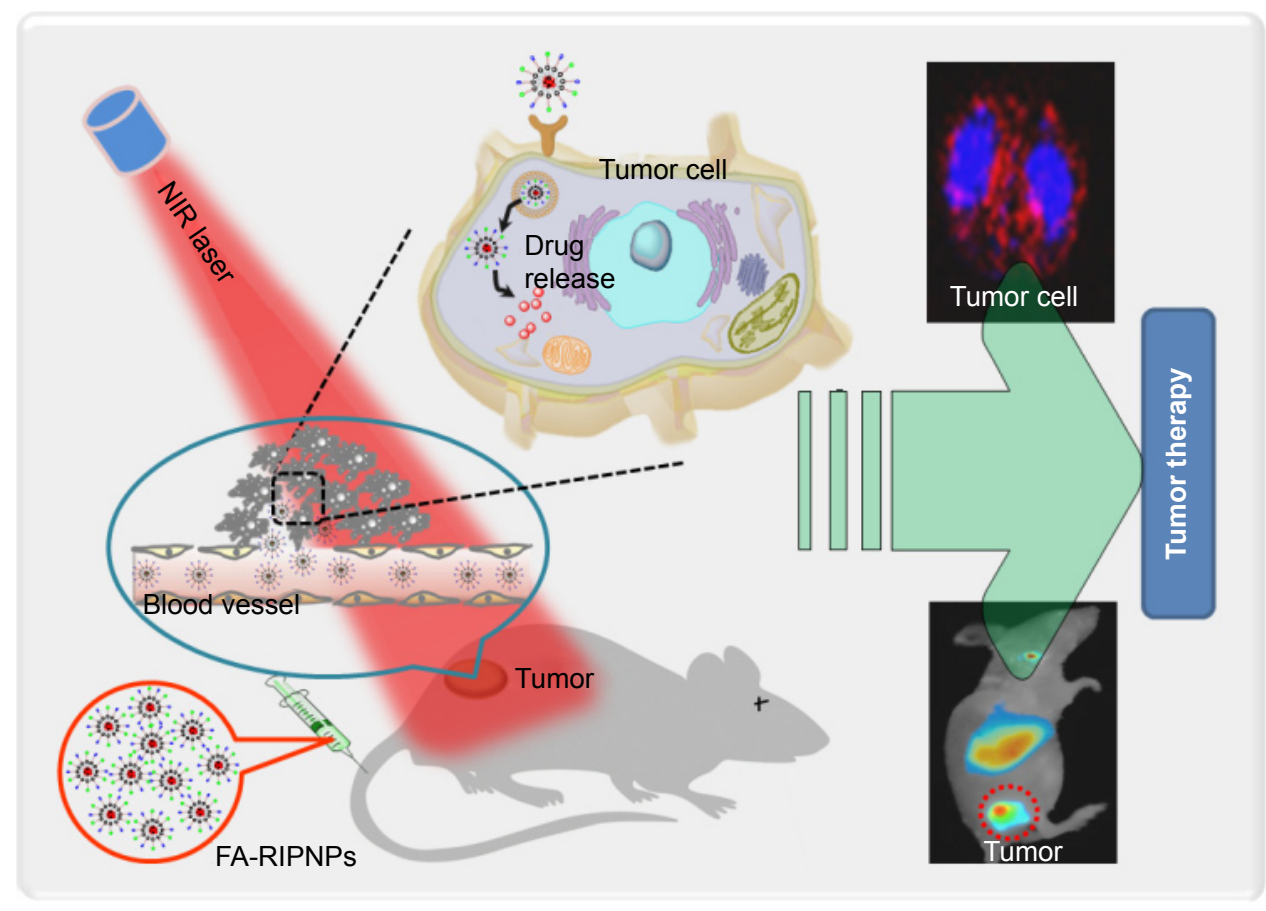

Scheme I Schematic representation of FA-RIPNPs as NIR nanoprobes.

Note: Schematic representation of FA-RIPNP use for tumor-targeted imaging and drug delivery in vitro and in vivo.

Abbreviations: NIR, near-infrared; FA, folic acid; RSV, resveratrol; ICG, indocyanine green; PLGA, poly(D,L-lactide-co-glycolide); NPs, nanoparticles; FA-RIPNPs, FA-RSV/ ICG-PLGA-lipid NPs.

(MEM), were purchased from Hyclone (Logan, UT, USA). All other chemicals and reagents were purchased from Sigma Aldrich.

\section{Preparation of FA-RIPNPs}

First, amino-ICG (ICG-NH ${ }_{2}$ ) was prepared following a literature procedure and was conjugated to DSPE-PEG ${ }_{2 \mathrm{k}}-\mathrm{COOH}$ via formation of an amide bond in the presence of EDC and NHS. ${ }^{20,37,38}$ The mixture was allowed to react for $48 \mathrm{~h}$ under nitrogen atmosphere at $25^{\circ} \mathrm{C}$. The reaction mixture was then dialyzed against deionized water for $3 \mathrm{~d}$ to remove the chemical catalysts and to obtain purified DSPE-PEG2k-ICG. Then, FA-RIPNPs were synthesized by a facile self-assembly-andnanoprecipitation method. ${ }^{14,39,40}$ Briefly, RSV (dissolved in dimethyl sulfoxide) was mixed with PLGA (dissolved in dichloromethane). Afterward, the PLGA/RSV solution was added dropwise into ethanol-aqueous solutions (4 wt\%) of lecithin/DSPE-PEG2k-ICG (4:1) and lecithin/DSPEPEG2k-FA (4:1) under gentle stirring, followed by vigorous vortexing for $5 \mathrm{~min}$ and gentle stirring for $4 \mathrm{~h}$ at $25^{\circ} \mathrm{C}$. To remove the nonencapsulated drug and organic solvents, the mixture was filtered and washed 3 times with deionized water using a Millipore ultrafiltration centrifuge tube. As a control, nanoparticles without FA molecules were prepared using the same method, in which DSPE-PEG 2,000 -FA was replaced by
DSPE-PEG ${ }_{2,000}-\mathrm{COOH}$. The purified FA-RIPNPs were stored at $4^{\circ} \mathrm{C}$ before use.

\section{Characterizations}

The morphology of the nanoparticles was studied by transmission electron microscopy (TEM; Tecnai ${ }^{\mathrm{TM}}$; FEI, Hillsboro, OR, USA) and atomic force microscopy (AFM; BioScope Catalyst ${ }^{\mathrm{TM}}$; Bruker, Santa Barbara, CA, USA). The size distribution and zeta potentials were measured by a Zetasizer Nano ZS system (Malvern Instruments, Malvern, UK). Absorption spectra were acquired using an ultravioletvisible (UV-Vis) spectrophotometer (Shimadzu UV-2550; Shimadzu Corporation, Kyoto, Japan). Fluorescence spectra were recorded using a fluorescence spectrometer (F900; Edinburgh Instruments Ltd, Livingston, UK). Fluorescence images of cells and living mice were acquired using confocal laser scanning microscopy (CLSM; FV1200; Olympus, Tokyo, Japan) and a commercial Maestro ${ }^{\mathrm{TM}}$ in vivo imaging system (CRi Inc, Woburn, MA, USA), respectively.

\section{Drug loading and release}

The encapsulation of RSV was carried out via self-assembly of RSV, PLGA and lipid, described in the "Preparation of FA-RIPNPs" section. The encapsulation efficiency (EE) of RSV was calculated using a UV-Vis spectrometer and 
the equations are listed below. A drug release study was conducted via a dynamic dialysis technique at $4^{\circ} \mathrm{C}$ and $37^{\circ} \mathrm{C}$ at pH 5.0 and pH 7.4, respectively. The drug concentration was calculated using a standard calibration curve. Cumulative release (CR) of RSV versus time was calculated according to the following equation:

$$
\mathrm{EE}(\%)=\frac{W_{1}}{W_{2}} \times 100 \%
$$

$W_{1}$ represents the weight of RSV in FA-RIPNPs, and $W_{2}$ is the weight of RSV added.

$$
\mathrm{CR}(\%)=\frac{W_{\mathrm{a}}}{W_{\mathrm{b}}} \times 100 \%
$$

$W_{\mathrm{a}}$ represents the amount of RSV released, and $W_{\mathrm{b}}$ is the total RSV present in FA-RIPNPs.

\section{In vitro cellular uptake}

Human glioma U87 cells were purchased from the Shanghai Institute of Cell Biology, Chinese Academy of Sciences, Shanghai, China, and were cultured in MEM supplemented with $10 \% \mathrm{FBS}$ and $1 \%$ penicillin-streptomycin solution in a humidified $5 \% \mathrm{CO}_{2}$ atmosphere at $37^{\circ} \mathrm{C}$.

For cellular uptake, U87 cells $\left(1 \times 10^{5}\right.$ cells $\left./ \mathrm{mL}, 0.5 \mathrm{~mL}\right)$ were seeded in $20 \mathrm{~mm}$ Petri dishes and were cultured for $24 \mathrm{~h}$ in an incubator $\left(5 \% \mathrm{CO}_{2}, 37^{\circ} \mathrm{C}\right)$. Next, free ICG, RIPNPs, FA-RIPNPs with free FA and FA-RIPNPs at the same concentration as ICG were added to the U87 cells and were subsequently incubated for $4 \mathrm{~h}$. Subsequently, the cells were gently washed with PBS three times, fixed with 4\% paraformaldehyde at room temperature for $15 \mathrm{~min}$ and stained with DAPI solution $(0.2 \mathrm{mg} / \mathrm{mL})$ for $10 \mathrm{~min}$. The fluorescence signals of the cells were monitored using CLSM.

\section{In vitro cytotoxicity and apoptosis detection}

U87 cells $\left(1 \times 10^{4}\right.$ cells per well $)$ were seeded in 96 -well plates and were incubated for $24 \mathrm{~h}$. Then, the cells were treated with ICG-PLGA-lipid NPs and FA-ICG-PLGA-lipid NPs at concentrations of 5, 10, 50 and $100 \mu \mathrm{g} / \mathrm{mL}$, as well as free RSV, RIPNPs and FA-RIPNPs at the same RSV concentration of $10,20,30$ and $50 \mu \mathrm{g} / \mathrm{mL}$ for $24 \mathrm{~h}$. Next, the old media in all wells were discarded and replaced with fresh media containing 10\% Cell Counting Kit-8 (Dojindo Laboratories, Kumamoto, Japan) solution. After incubation for $30 \mathrm{~min}$, the relative cell viability was assessed using a microplate reader
(BioTek, Winooski, VT, USA) at an absorbance wavelength of $450 \mathrm{~nm}$. An Annexin V-fluorescein isothiocyanate (FITC)/ propidium iodide (PI) apoptosis detection kit (BestBio, Shanghai, China) was used to detect the cell apoptosis rate, which was analyzed by flow cytometry (FCM). Briefly, U87 cells were incubated in 6-well plates $\left(1 \times 10^{4}\right.$ cells per well) for $24 \mathrm{~h}$. Free RSV, RIPNPs and FA-RIPNPs at the same RSV concentration of $30 \mu \mathrm{g} / \mathrm{mL}$ were administered to the cells, and these were incubated for $24 \mathrm{~h}$. According to the manufacturer's instructions, the cells were collected, washed, stained with Annexin V-FITC/PI and analyzed by FCM.

\section{Blood circulation study}

Twenty BALB/c nude mice were randomly divided into four groups (five mice per group). RSV, ICG, RIPNPs and FA-RIPNPs were intravenously administered. Blood samples $(\sim 100 \mu \mathrm{L})$ were drawn from the orbit $6,18,30$ and $42 \mathrm{~min}, 1,3,5,7,12$ and $24 \mathrm{~h}$ following intravenous administration. Each blood sample was dissolved in $900 \mu \mathrm{L}$ of lysis buffer $\left(10 \mathrm{mmol} / \mathrm{L} \mathrm{Na}_{2} \mathrm{HPO}_{4}\right.$, pH 7.5, $5 \mathrm{mmol} / \mathrm{L}$ EDTA, $100 \mathrm{mmol} / \mathrm{L} \mathrm{NaCl}, 1 \%$ Triton, $0.5 \%$ sodium deoxycholate, $0.1 \%$ SDS). The concentration of RSV, ICG, FA-RIPNPs and RIPNPs in the blood was determined by the RSV and ICG absorbance spectra of each solubilized blood sample using a UV-Vis spectrometer. The sample concentrations are shown as the percentage of injected dose per gram of tissue (ID $\% / g)$.

\section{Tumor model and in vivo imaging study}

$\mathrm{BALB} / \mathrm{c}$ nude mice (6-8 wk old) were purchased from Charles River Laboratories (Beijing, People's Republic of China). All safety and experimental procedures were performed in accordance with the Guidelines for the Care and Use of Laboratory Animals of Capital Medical University and were approved by the Ethics Committee of Capital Medical University (GJCY150908-5; August 12, 2015).

U87 glioma xenograft models were established by subcutaneously injecting U87 cells (in $150 \mu \mathrm{L}$ PBS) into the back of mice $\left(3 \times 10^{6}\right.$ cells per mouse). The mice were used for further experimental procedures when the overall tumor volume reached $100 \mathrm{~mm}^{3}$.

Fluorescence images were collected 0, 1, 6, 24, 48 and $72 \mathrm{~h}$ postinjection of ICG, RIPNPs and FA-RIPNPs at an excitation wavelength of $735 \mathrm{~nm}$. The fluorescence images of excised tumor slices $6 \mathrm{~h}$ postinjection of ICG, RIPNPs and FA-RIPNPs were also collected. The tumor-bearing mice were sacrificed $24 \mathrm{~h}$ postinjection of ICG, RIPNPs and FA-RIPNPs, and the sample contents in the major tissues, including heart, liver, spleen, lungs, kidneys and tumor, 
were also evaluated using a fluorescence spectrometer. Briefly, the major tissues were dissected, weighed and dissolved in lysis buffer. The concentration of RSV, ICG and FA-RIPNPs in these samples was determined by the RSV and ICG fluorescence of each solubilized sample.

\section{In vivo anticancer efficacy}

For in vivo anticancer efficacy, the U87 tumor-bearing mice were randomly divided into four groups (five mice per group) and intravenously injected with saline (control), free RSV, RIPNPs and FA-RIPNPs, respectively. The tumor volume and body weight of mice were recorded every $3 \mathrm{~d}$, and the tumor size was calculated as follows: volume $(V)=$ length $\times$ width $^{2} / 2$. The relative tumor volumes were calculated as $V / V_{0}$, where $V_{0}$ represents the tumor volume before the treatment was started.

\section{Histology and blood examination}

After about 1 month following treatment with FA-RIPNPs, the mice were sacrificed and the major organs, including heart, liver, spleen, lungs and kidneys, were collected. The organs were fixed in $4 \%$ paraformaldehyde overnight and dehydrated in $25 \%$ sucrose. Next, the fixed tissues were sectioned into $5 \mu \mathrm{m}$-thick slices, stained with hematoxylin and eosin (H-E, Sigma), according to the manufacturer's instructions, and studied using an inverted microscope to observe any histological alterations.

In order to record the complete blood counts, ${ }^{41,42}$ healthy $\mathrm{BALB} / \mathrm{c}$ mice were intravenously injected with $200 \mu \mathrm{L}$ FARIPNPs at a dose of $15 \mathrm{mg} / \mathrm{kg}$. Saline-treated mice $(200 \mu \mathrm{L})$ were used as the control group. The mice were sacrificed before blood collection $(0.5 \mathrm{~mL})$, and complete blood count evaluations at 1, 7, 14 and $28 \mathrm{~d}$ postinjection of FA-RIPNPs (five mice per group) were carried out at the Beijing Tiantan Hospital of Capital Medical University, Beijing, People's Republic of China.

\section{Statistical analysis}

Data are shown as mean \pm SD. Two-tailed Student's $t$-test was used to analyze the statistical significance of the differences between two groups. The differences were considered significant for $* P<0.05$ and highly significant for $* * P<0.01$.

\section{Results and discussion Preparation and characterization of FA-RIPNPs}

The FA-RIPNPs were prepared by a facile self-assemblyand-nanoprecipitation method, as shown in Figure 1A.

A
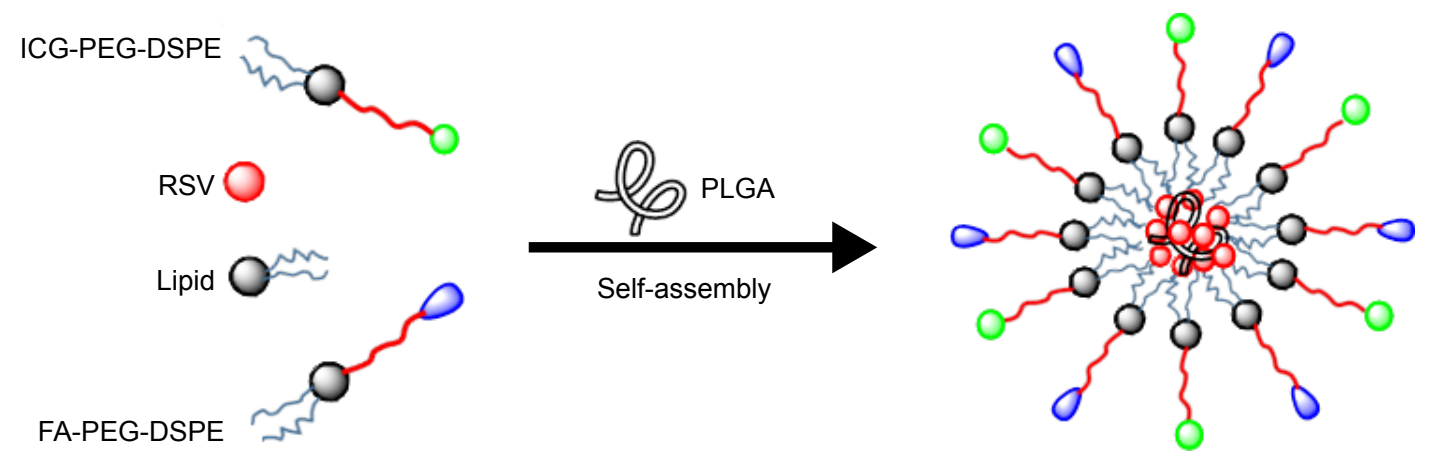

FA-RIPNPs

B

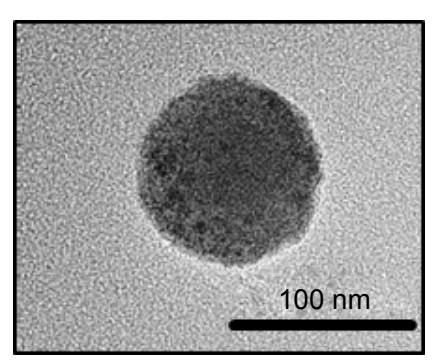

C

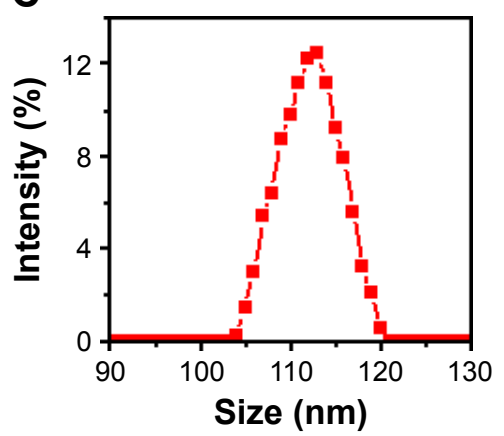

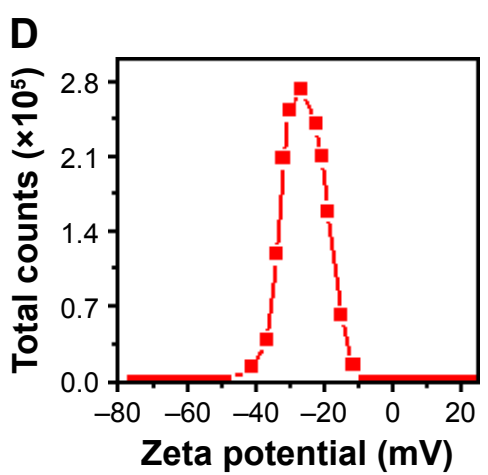

Figure I Preparation and morphology of FA-RIPNPs.

Notes: (A) Schematic representation of synthesis by self-assembly, (B) TEM image, (C) size distribution and (D) zeta potential distribution of FA-RIPNPs.

Abbreviations: TEM, transmission electron microscope; FA, folic acid; RSV, resveratrol; ICG, indocyanine green; PLGA, poly(D,L-lactide-co-glycolide); NPs, nanoparticles; FA-RIPNPs, FA-RSV/ICG-PLGA-lipid NPs; PEG-DSPE, I,2-distearoyl-sn-glycero-3 phosphoethanolamine-N-[(polyethylene glycol)-2000]. 
PLGA polymer encapsulated RSV to form a core. DSPEPEG $_{2,000}$-FA and DSPE-PEG ${ }_{2,000}$-ICG self-assembled around the core to form a hydrophilic monolayer and stabilized the entire nanoparticle. Figure 1B shows that FA-RIPNPs exhibited a well-defined spherical shape, which corresponded to the results of AFM analysis (Figure S1). The diameter (Figure 1C) and zeta potential (Figure 1D) of the FA-RIPNPs were determined to be $104.5-121.1 \mathrm{~nm}$ and $-8.4 \mathrm{mV}$ to $-47.3 \mathrm{mV}$, respectively.

UV-Vis spectra were obtained to confirm the presence of FA-RIPNPs. After dialysis against deionized water to remove the chemical catalysts, free ICG and RSV, the dialysate of the dialysis bag was confirmed to have no ICG and RSV by using a UV-Vis spectrophotometer. As shown in Figure 2A, FA-RIPNPs simultaneously featured absorbance peaks for ICG at $780 \mathrm{~nm}$ and RSV at $304 \mathrm{~nm}$, indicating the presence of both ICG and RSV in FA-RIPNPs. The ICG$\mathrm{NH}_{2}$ prepared here exhibited a similar absorbance spectrum as ICG (Figure S2). In addition, FA-RIPNPs showed ICG $(\sim 800 \mathrm{~nm})$ and RSV ( 400 nm) fluorescence signals upon excitation with a $765 \mathrm{~nm}$ and $325 \mathrm{~nm}$ laser, which proves to be in agreement with the fluorescence spectra of ICG and RSV (Figure 2B and C). We found that ICG-NH $\mathrm{N}_{2}$ also features an $\sim 800 \mathrm{~nm}$ fluorescence signal at $765 \mathrm{~nm}$ excitation wavelength (Figure S3). These results demonstrate that both ICG and RSV maintain their optical properties after conjugation to FA-RIPNPs.

Figure $3 \mathrm{~A}$ shows the change in EE of RSV within FARIPNPs upon increasing the RSV concentration. The maximum RSV and ICG (figure not shown) EE of FA-RIPNPs obtained was $65.6 \% \pm 4.7 \%$ and $38.4 \% \pm 4.8 \%$, respectively. Furthermore, as shown in Figure 3B, FA-RIPNPs exhibited the highest RSV release rate of $78.2 \% \pm 4.1 \%$ after $36 \mathrm{~h}$ at $37^{\circ} \mathrm{C}$ at $\mathrm{pH} 5.0$, compared to the release rate obtained at different conditions, including $37^{\circ} \mathrm{C}$ at $\mathrm{pH} 7.4,4^{\circ} \mathrm{C}$ at $\mathrm{pH} 7.4$ and $4^{\circ} \mathrm{C}$ at $\mathrm{pH} 5.0$, while the ICG release rate proved to be less than $\sim 20 \%$ in all test conditions (Figures 3B, S4-S6). This finding may be due to the weak noncovalent interactions of RSV and the covalent conjugation of ICG, leading to a high release rate of RSV and a reduced release rate of ICG. ${ }^{20}$
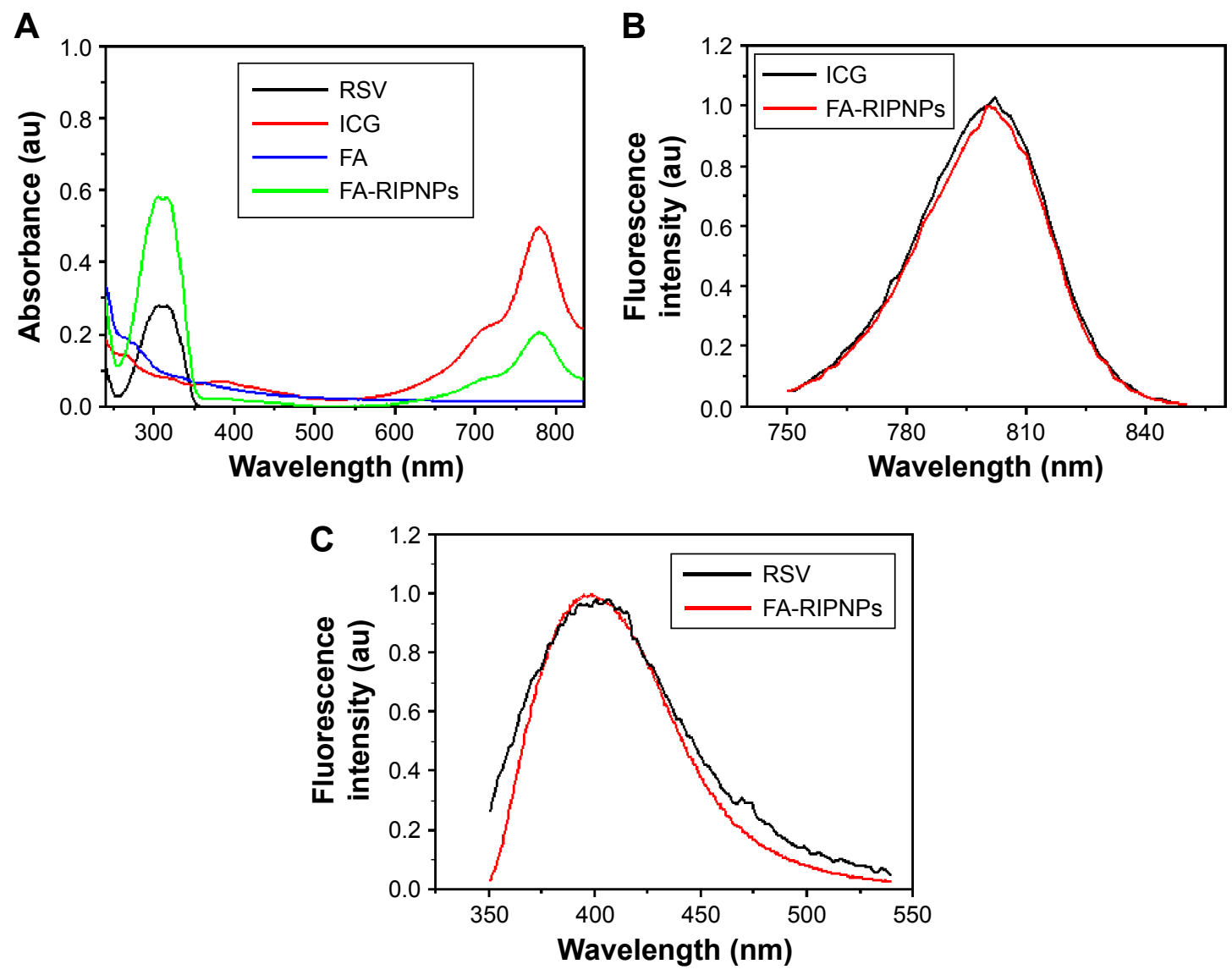

Figure 2 Spectral characterizations.

Notes: (A) Absorbance spectra of free RSV, free ICG, FA and FA-RIPNPs. (B) Normalized fluorescence spectra of free ICG and FA-RIPNPs at the same concentration of ICG. (C) Normalized fluorescence spectra of free RSV and FA-RIPNPs at the same concentration of RSV.

Abbreviations: RSV, resveratrol; ICG, indocyanine green; FA, folic acid; PLGA, poly(D,L-lactide-co-glycolide); NPs, nanoparticles; FA-RIPNPs, FA-RSV/ICG-PLGA-lipid NPs. 
A

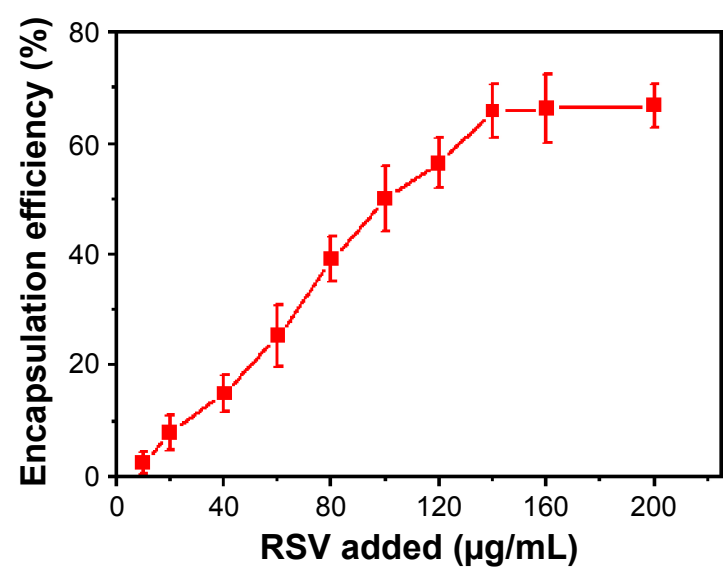

B

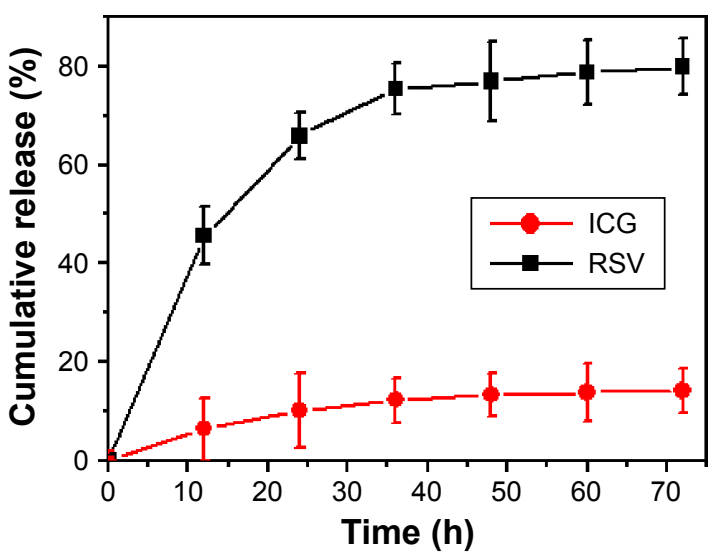

Figure 3 Loading and release of FA-RIPNPs.

Notes: (A) RSV encapsulation efficiency as a function of added RSV concentrations, $n=6$. (B) In vitro release profiles of ICG and RSV from FA-RIPNPs at pH 5.0 and $37^{\circ} \mathrm{C}, \mathrm{n}=6$.

Abbreviations: RSV, resveratrol; ICG, indocyanine green; FA, folic acid; PLGA, poly(D,L-lactide-co-glycolide); NPs, nanoparticles; FA-RIPNPs, FA-RSV/ICG-PLGA-lipid NPs.

As has already been shown, the $\mathrm{pH}$ environment in the blood stream is $\mathrm{pH} 7.4$ and, in tumor tissue, the $\mathrm{pH}$ often proves to be slightly acidic. These results are beneficial for the application of FA-RIPNPs in tumor therapy and imaging. ${ }^{43-45}$

The excellent optical properties, including NIR fluorescence and absorbance, render ICG a good candidate for use as an imaging probe in clinical diagnosis. However, numerous disadvantages, including poor solubility in aqueous media and low photostability, generally limit biomedical applications. ${ }^{16}$ The applicability of insoluble RSV is also limited by its poor stability in aqueous solutions ${ }^{29}$ Figure 4A and $\mathrm{B}$ shows the fluorescence stability of ICG and FARIPNPs in aqueous solution at $4^{\circ} \mathrm{C}$ and $37^{\circ} \mathrm{C}$, respectively. After $4 \mathrm{wk}$ of storage at $4{ }^{\circ} \mathrm{C}$ and $37^{\circ} \mathrm{C}$, the ICG fluorescence intensity of FA-RIPNPs remained $89.9 \%$ and $85.7 \%$ of the initial intensities, while the fluorescence of ICG dropped rapidly to $0.23 \%$ and $0.08 \%$ of its initial intensity. The corresponding images of ICG in aqueous solution showed an enhanced aggregation from $4^{\circ} \mathrm{C}$ to $37^{\circ} \mathrm{C}$, while FA-RIPNPs in aqueous solution indicate a consistent stability (insets in Figure 4A and B). The average size of FA-RIPNPs in other media, including water, MEM, PBS and FBS, features almost no changes over $4 \mathrm{wk}$ (Figure $4 \mathrm{C}$ ), providing further evidence for the high colloidal stability of FA-RIPNPs, most likely due to the PEG shell around the PLGA core. According to published literature reports, RSV naturally exists as two isomers, cis and trans, which feature absorbance peaks at $280 \mathrm{~nm}$ and $304 \mathrm{~nm}$, respectively. ${ }^{28}$ The trans-isomer exhibits higher bioactivity and proves to be more stable than the $c i s$-isomer. Furthermore, the $c i s$-isomer is known to be easily formed from the trans-isomer via heat induction. ${ }^{29,46}$ As shown in Figure 4D and the inset, the ratio of the absorbance value $(A)_{304 \mathrm{~mm}} / \mathrm{A}_{280 \mathrm{~nm}}$ remained almost unchanged over $4 \mathrm{wk}$ at $37^{\circ} \mathrm{C}$, indicating that the RSV portion in FA-RIPNPs was indeed the trans-isomer that could be protected by PLGA and lipid encapsulation at $37^{\circ} \mathrm{C}$. The results obtained suggest that the covalently conjugated ICG and encapsulated RSV were more stable than the free ICG and RSV species, most notably due to the encapsulation protecting ICG and RSV from direct environmental influences, such as heat or light. This allows for prolonged storage times and ultimately improves the applicability of this material as a theranostic agent.

\section{In vitro targeting fluorescence imaging}

A schematic representation of FA-mediated targeted fluorescence imaging is shown in Figure 5A. FA-RIPNPs are able to efficiently target cells and internalize within cells likely via FA receptor-mediated endocytosis (RME), going through a series of processes: nanoparticles first stick to the cell membrane, the membrane then wraps the nanoparticles and, finally, the pinching off occurs (particle-lipid complex detaches from the membrane and enters the cell). ${ }^{47}$ To study the cell uptake of FA-RIPNPs, FA receptor (FR)-containing U87 cells were incubated with FA-RIPNPs for $4 \mathrm{~h}$, and the cell nuclei were stained with DAPI. As shown in Figure 5B, a strong red fluorescence (ICG signal) was observed in the perinuclear region in the U87 cells treated with FA-RIPNPs, indicating that a sufficient amount of FA-RIPNPs enter the cells. As a control, U87 cells after treatment with free ICG and RIPNPs showed very little red fluorescence in the cytoplasm (Figure 5B). Interestingly, upon incubation with FA-RIPNPs and free FA, the U87 cells also showed 

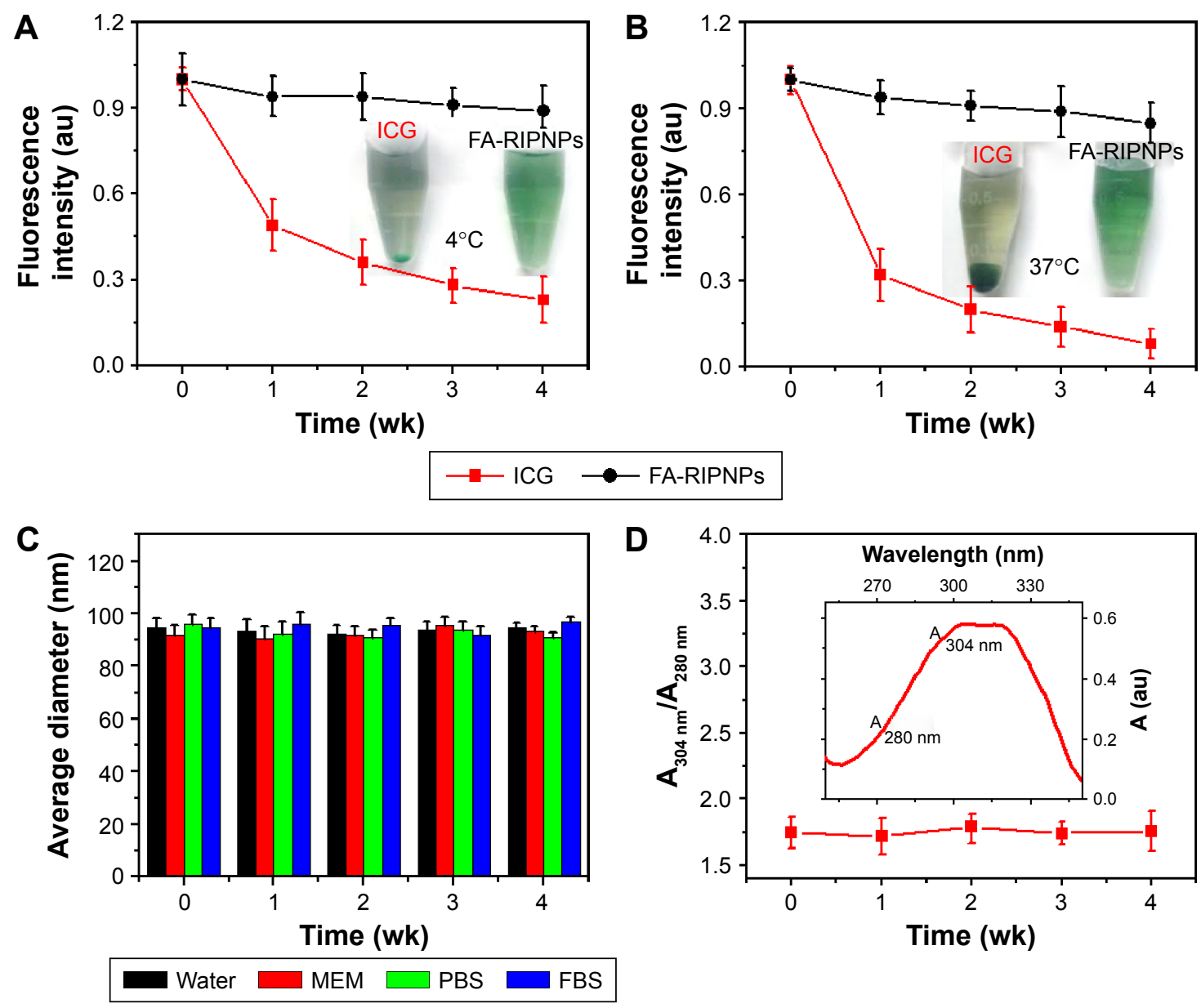

Figure 4 Fluorescence stability and stability in water of FA-RIPNPs.

Notes: ICG fluorescence stability of free ICG and FA-RIPNPs in aqueous solution at (A) $4^{\circ} \mathrm{C}$ and (B) $37^{\circ} \mathrm{C}$ over 4 wk. Insets show photos of free ICG and FA-RIPNPs in aqueous solution after $4 \mathrm{wk}$ of storage at $4^{\circ} \mathrm{C}$ and $37^{\circ} \mathrm{C}$, respectively. (C) Colloidal stability test of FA-RIPNPs in different media, including water, MEM, PBS and FBS. (D) Change in ratio of absorbances at $304 \mathrm{~nm}$ and $280 \mathrm{~nm}\left(\mathrm{~A}_{304 \mathrm{~nm}} / \mathrm{A}_{280 \mathrm{~nm}}\right)$ of the FA-RIPNPs over 4 wk at $37^{\circ} \mathrm{C}$. Inset shows the absorbance spectrum of FA-RIPNPs.

Abbreviations: RSV, resveratrol; ICG, indocyanine green; FA, folic acid; PLGA, poly(D,L-lactide-co-glycolide); NPs, nanoparticles; FA-RIPNPs, FA-RSV/ICG-PLGA-lipid NPs; MEM, minimum essential medium; PBS, phosphate-buffered saline; FBS, fetal bovine serum.

insufficient red fluorescence, likely due to the FRs on the U87 surface being blocked by free FA. ${ }^{48}$ These results demonstrate the high internalization of FA-RIPNPs, with excellent positive targeting capabilities by U87 cells.

\section{In vitro cytotoxicity and apoptosis detection}

The cytotoxicity of ICG-PLGA-lipid NPs and FA-ICGPLGA-lipid NPs, as the carriers of RSV and as NIR probes, in the concentration range of $0-100 \mu \mathrm{g} / \mathrm{mL}$ was examined in vitro. As shown in Figure 6A, U87 cells were incubated with the two nanoparticle species, and the cell viability was maintained above $90 \%$, suggesting that they do not induce acute cytotoxicity and are highly cell compatible.

Furthermore, we examined the anticancer effect of FA-RIPNPs in vitro. As shown in Figure 6B, treatment with free RSV and RIPNPs at RSV concentrations ranging between $0 \mu \mathrm{g} / \mathrm{mL}$ and $50 \mu \mathrm{g} / \mathrm{mL}$ decreased cell viability in a dose-dependent manner. However, FA-RIPNPs at the same RSV concentration caused a considerably higher U87 cell death rate $(P<0.05)$. The cytotoxic enhancements of FARIPNPs could be attributed to the more efficient FA RME of FA-RIPNPs in U87 cells and the high RSV release rate. In addition, the cell death type of U87 cells induced by FARIPNPs was investigated by an Annexin V-FITC/PI doublestaining kit and was further analyzed by FCM. According to the literature, the Q2 + Q4 region in the representative dot plots from FCM are defined as apoptotic cells..$^{49,50}$ As shown in Figure 6C-F, the U87 cells treated with FA-RIPNPs (30 $\mu \mathrm{g} / \mathrm{mL} \mathrm{RSV}$ ) for $24 \mathrm{~h}$ demonstrated an $82.7 \%$ apoptosis rate. This rate was much higher than that of free RSV (41.5\%) and RIPNPs (47.1\%) at the same RSV concentration, consistent with the results shown in Figure 6B. U87 cells treated with blank NPs with dye and FA (FA-ICG-PLGA-lipid NPs) 
A

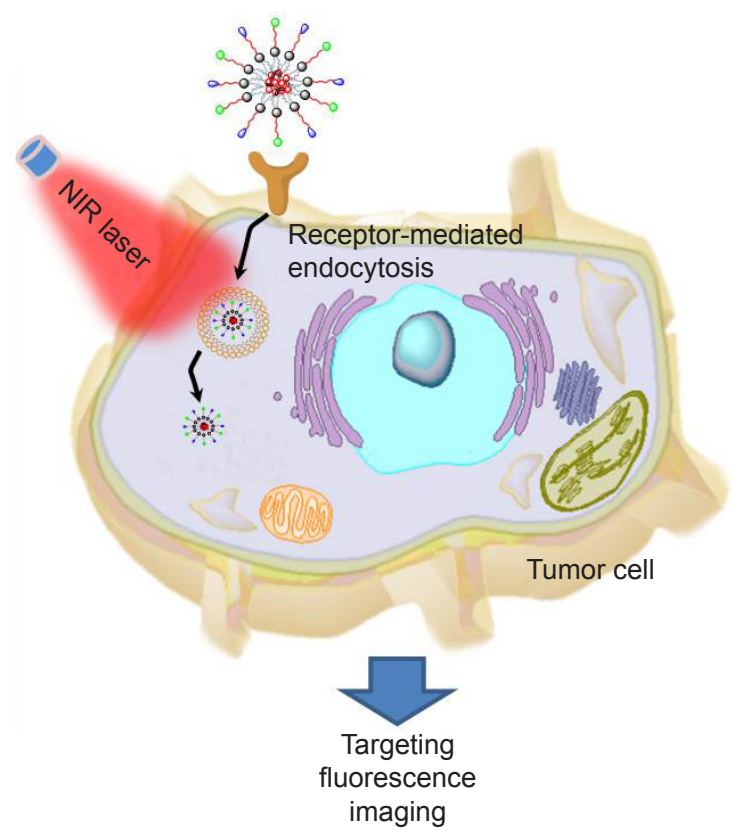

B
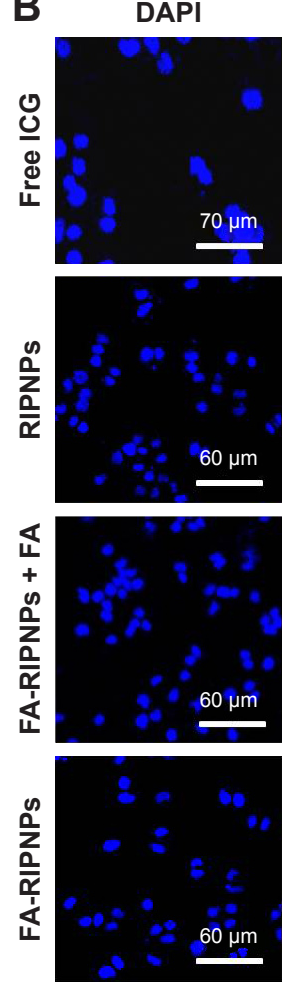

ICG
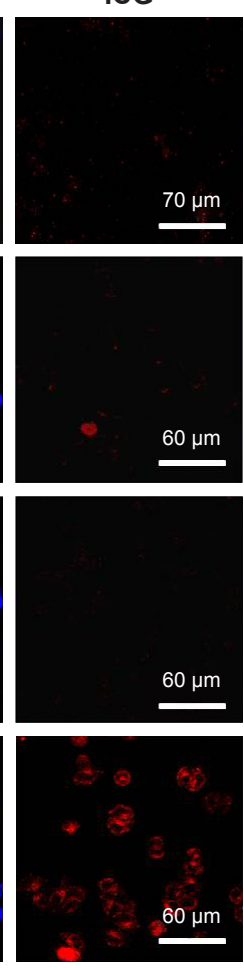

Overlay
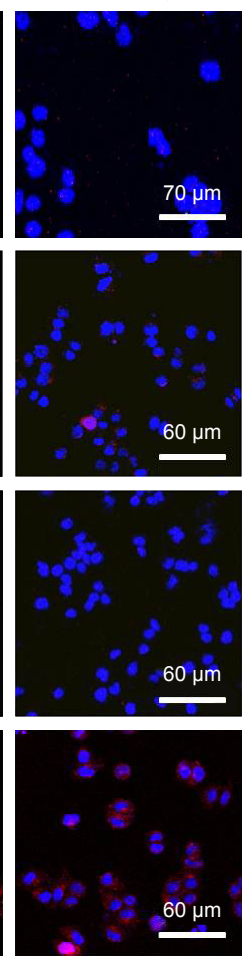

Figure 5 Cellular uptake of the study compounds.

Notes: (A) Schematic representation of cellular uptake. (B) Fluorescence images of U87 cellular uptake of free ICG, RIPNPs, FA-RIPNPs with FA blocking and FA-RIPNPs after $4 \mathrm{~h}$ of incubation.

Abbreviations: NIR, near-infrared; RSV, resveratrol; ICG, indocyanine green; FA, folic acid; PLGA, poly(D,L-lactide-co-glycolide); NPs, nanoparticles; FA-RIPNPs, FA-RSV/ ICG-PLGA-lipid NPs; DAPI, 4',6-diamidino-2-phenylindole.

showed lower apoptosis rate, which was similar to that of the control group (Figure S7).

\section{Blood circulation}

Prolonged circulation of nanoparticles in the bloodstream is essential to improve the probability for entering the tumor and accumulation therein. As can be seen in Figure 7, free RSV and ICG were quickly removed from the blood circulation, and their $t_{1 / 2}$ was assessed to be $1.02 \pm 0.05 \mathrm{~h}$ and $0.18 \pm 0.02 \mathrm{~h}$, respectively. However, FA-RIPNPs and RIPNPs displayed a considerably longer blood circulation time at $t_{1 / 2}=7.1 \pm 0.84 \mathrm{~h}$ and $t_{1 / 2}=6.8 \pm 1.1 \mathrm{~h}$, respectively. This result indicates that PLGA-and-lipid encapsulation could delay the elimination of ICG as well as RSV and therefore prolongs the circulation time in the bloodstream, ${ }^{51}$ a feature that may have a positive effect on in vivo therapy and imaging.

\section{In vivo targeting fluorescence imaging and tissue biodistribution}

A high tumor accumulation of nanodrugs and a quick clearance rate are essential features for the clinical applicability of such materials. Noninvasive in vivo fluorescence imaging was used to evaluate the tumor targeting efficacy and biodistribution characteristics of FA-RIPNPs in subcutaneous U87 tumor-bearing mice after intravenous injection of FA-RIPNPs. As depicted in Figure 8A, strong fluorescence signals were obtained in the entire body 5 min postinjection of free ICG, RIPNPs and FA-RIPNPs, whereas $6 \mathrm{~h}$ postinjection, more intense fluorescence signals were obtained in the tumor region for the FA-RIPNP group, compared to the ICG and RIPNP groups. This finding was further confirmed by the fluorescence images of the corresponding excised tumor slices as shown in Figure 8B. Figure 8C shows the change in fluorescence signal in the tumor region $48 \mathrm{~h}$ after injection of free ICG, RIPNPs and FA-RIPNPs. As expected, the fluorescence intensity in the tumor in the FA-RIPNP group remained very high for up to $48 \mathrm{~h}$. The signal intensity was obviously higher than that of free ICG and RIPNPs at all investigated time points. Additionally, a quantitative analysis of the ex vivo fluorescence of harvested tumors and major tissues, including heart, liver, spleen, lungs and kidneys, from euthanized mice $24 \mathrm{~h}$ postinjection was conducted as shown in Figure 8D. Strong fluorescence signals were detected in liver tissue in the groups of free ICG, RIPNPs and 

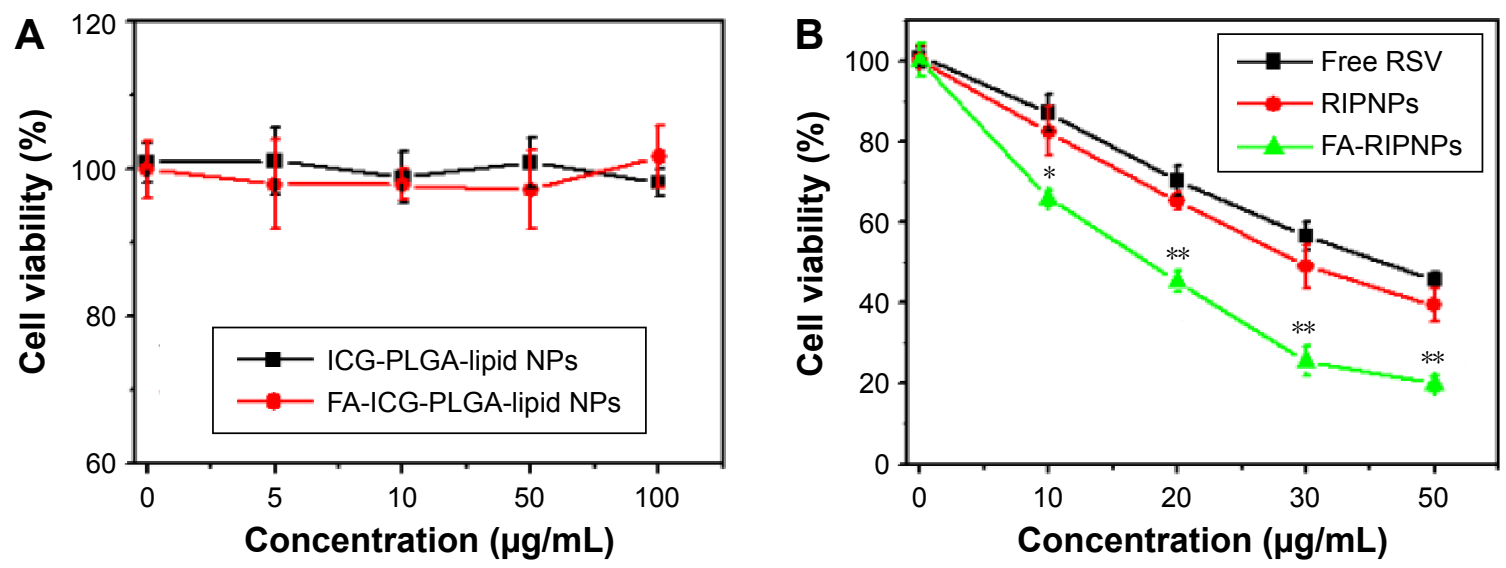

C

Control

D
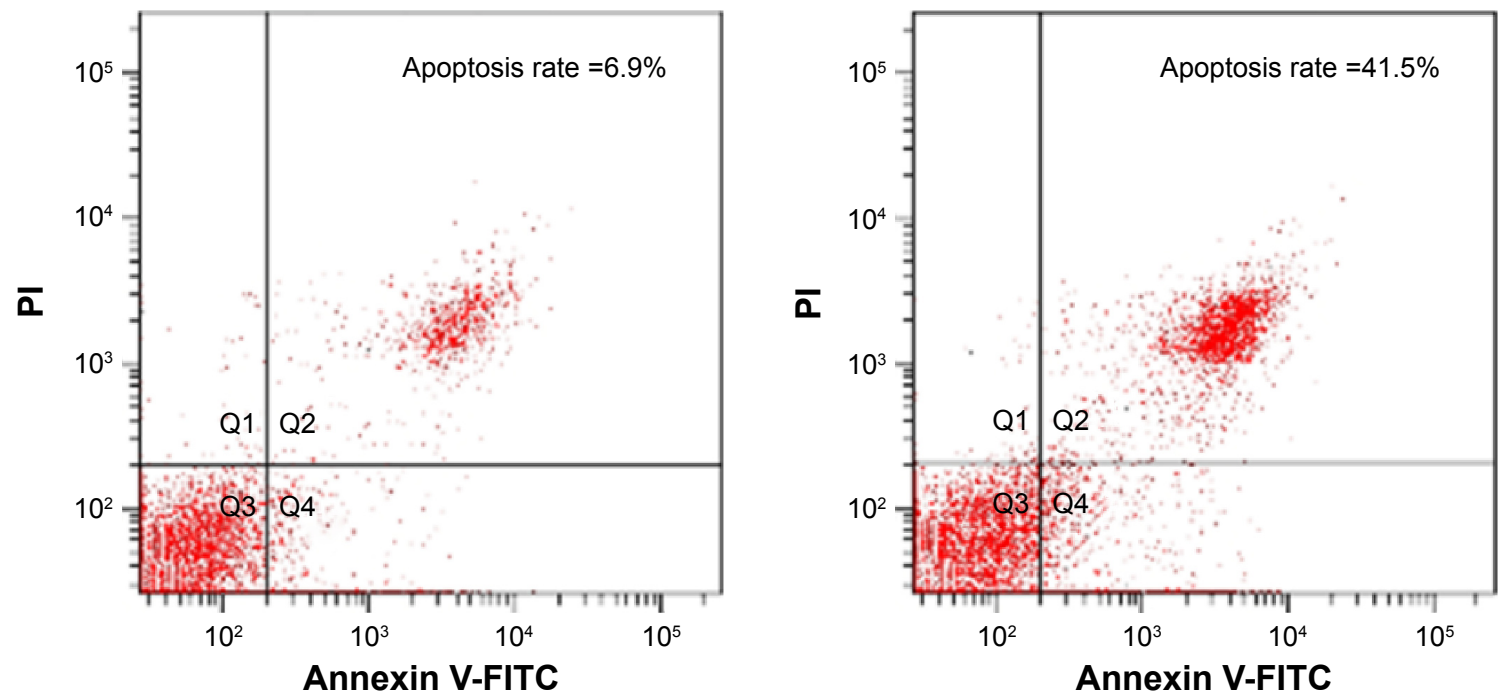

E

RIPNPs

$\mathbf{F}$

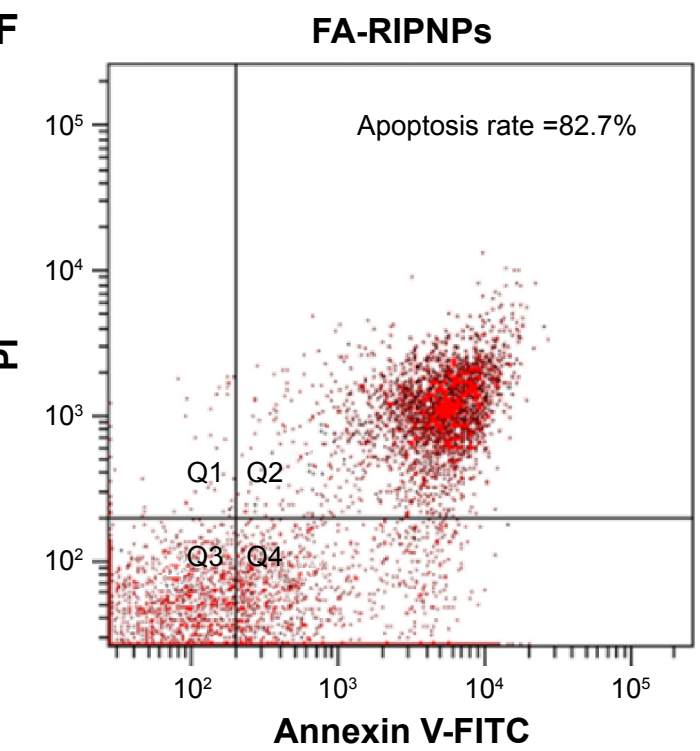

Figure 6 In vitro tumor therapy.

Notes: (A) Cytotoxicity of ICG-PLGA-lipid NPs and FA-ICG-PLGA-lipid NPs. (B) Cytotoxicity of free RSV, RIPNPs and FA-RIPNPs at the same concentration of RSV. $* P<0.05$, $* * P<0.01$. (C-F) Flow cytometry analysis of U87 cells treated with (C) saline, (D) free RSV, (E) RIPNPs and (F) FA-RIPNPs at the same concentration of RSV. The cells in Q2 + Q4 regions are defined as apoptotic cells.

Abbreviations: RSV, resveratrol; ICG, indocyanine green; FA, folic acid; PLGA, poly(D,L-lactide-co-glycolide); NPs, nanoparticles; FA-RIPNPs, FA-RSV/ICG-PLGA-lipid NPs; FITC, fluorescein isothiocyanate; $\mathrm{PI}$, propidium iodide. 


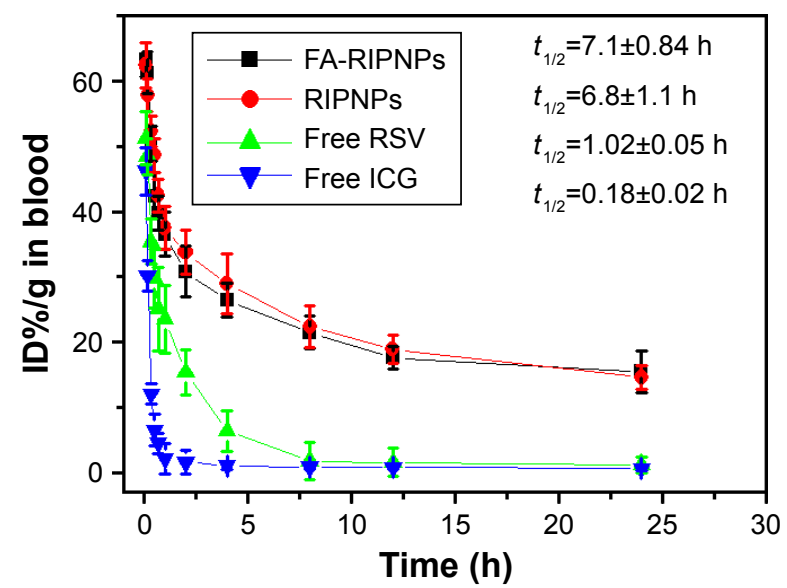

Figure 7 Blood circulation curves.

Notes: Blood circulation curves of free RSV, free ICG, RIPNPs and FA-RIPNPs in mice after intravenous injection determined by the ICG and RSV absorbances of diluted tissue lysate (the accumulation levels of ICG and RSV were calculated as ID\%/g, the percentage of the injected dose per gram of tissue).

Abbreviations: RSV, resveratrol; ICG, indocyanine green; FA, folic acid; PLGA, poly(D,L-lactide-co-glycolide); NPs, nanoparticles; FA-RIPNPs, FA-RSV/ICG-PLGAlipid NPs; ID, injected dose.
FA-RIPNPs, indicating that the main metabolic conversion of these compounds follows a hepatic pathway. Moreover, the FA-RIPNP group showed remarkably stronger fluorescence signals in the tumor than the RIPNPs and free ICG groups, approximately 2.8- and 12.6-fold higher respectively than the two latter groups. These data indicate that FA-RIPNPs do indeed feature a highly selective accumulation behavior in tumor tissue in vivo, likely due to the enhanced permeability and retention (EPR) characteristics of solid tumors and the FA RME effect. ${ }^{52}$

\section{In vivo anticancer efficacy}

Encouraged by the excellent in vitro anticancer efficacy and in vivo tumor-targeting behavior of FA-RIPNPs described, the anticancer efficacy of FA-RIPNPs was tested in U87 tumorbearing mice. As shown in Figure 9, no complete tumor growth suppression was observed in the control (saline)-, RSV- and RIPNP-treated mice at the same dose of RSV.
A

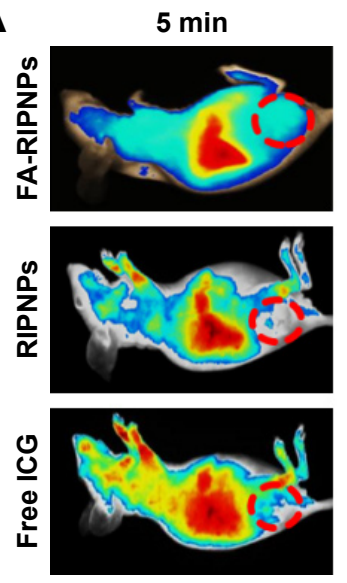

C

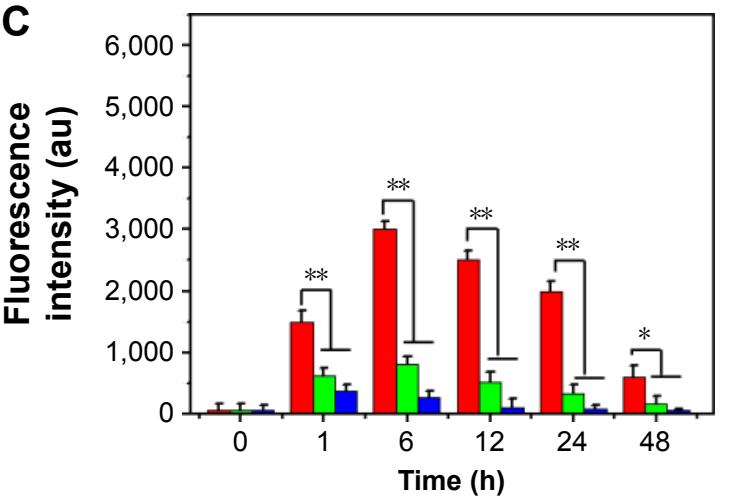

B
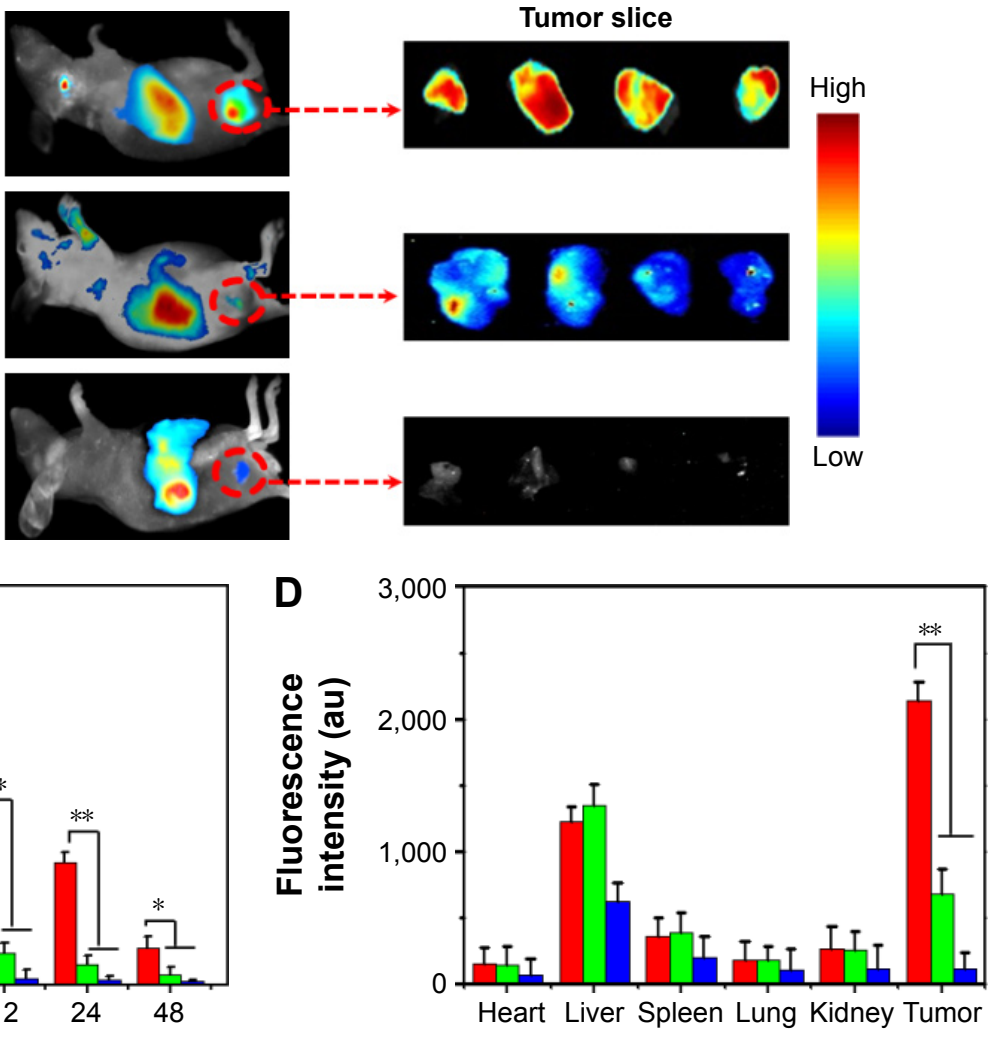

FA-RIPNPs $\square$ RIPNPs $\square$ Free ICG

Figure 8 In vivo tumor-targeted fluorescence images and biodistribution.

Notes: (A) Representative fluorescence images of U87 tumor-bearing mice after tail vein injection with free ICG, RIPNPs and FA-RIPNPs; and (B) corresponding tumor slices. The red dashed circles indicate the tumor region. (C) Quantitative in vivo analysis of the fluorescence signals of the tumor regions in free ICG-, RIPNP- and FARIPNP-treated mice as a function of injection time. $* P<0.05$, $* * P<0.01$. (D) ICG fluorescence signals of tumor and major organs, including heart, liver, spleen, lungs and kidneys. $* * P<0.01$.

Abbreviations: RSV, resveratrol; ICG, indocyanine green; FA, folic acid; PLGA, poly(D,L-lactide-co-glycolide); NPs, nanoparticles; FA-RIPNPs, FA-RSV/ICG-PLGA-lipid NPs. 


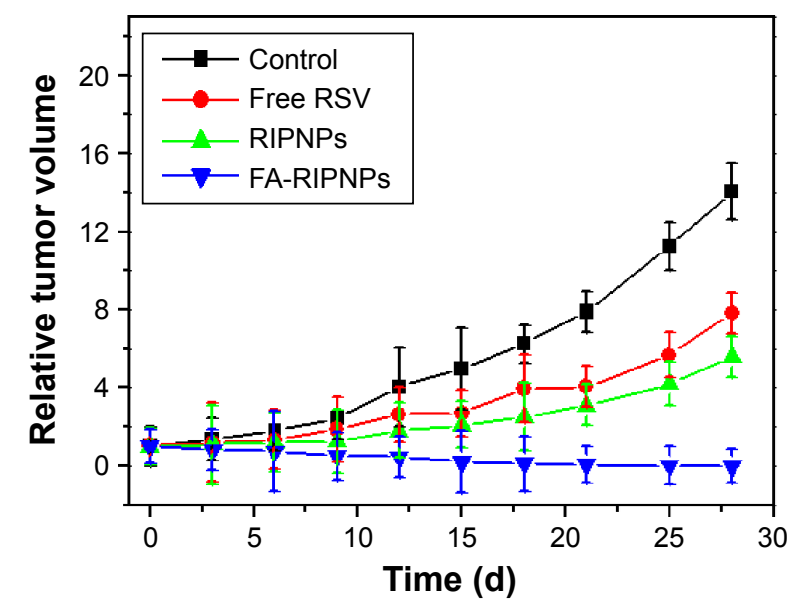

Figure 9 In vivo tumor therapy.

Notes: Relative tumor volumes of tumor-bearing mice after tail vein injection with control (saline), free RSV, RIPNPs and FA-RIPNPs.

Abbreviations: RSV, resveratrol; FA, folic acid; ICG, indocyanine green; PLGA, poly(D,L-lactide-co-glycolide); NPs, nanoparticles; FA-RIPNPs, FA-RSV/ICG-PLGAlipid NPs.

However, intravenous injection of FA-RIPNPs exhibited remarkable tumor growth suppression, and no tumor relapse was observed after about 1 month of treatment. This result indicates that FA-RIPNPs feature a powerful in vivo anticancer efficacy.

\section{Histology and blood examination}

Generally, the toxicity of nanoparticle agents is a crucial criterion in nanomedicine. After approximately 1 month of treatment, no significant body weight loss was observed in all groups (Figure 10A). The major organs of mice, including heart, liver, spleen, lungs and kidneys, in each group were collected, sliced and stained with H-E. However, none of the organs exhibited obvious histological lesions (Figure 10B).
To further evaluate the potential toxicity of FA-RIPNPs, complete blood counts were obtained from FA-RIPNPinjected ( $15 \mathrm{mg} / \mathrm{kg}$ ) healthy BALB/c mice 1, 7, 14 and $28 \mathrm{~d}$ postinjection. All parameters, including white blood cells, red blood cells, hemoglobin, hematocrit, mean corpuscular hemoglobin, mean corpuscular hemoglobin concentration, mean corpuscular volume, mean platelet volume and platelets, were shown to be within reference ranges for healthy BALB/c mice (Figure 10C). Taken in concert, FA-RIPNPs exhibited high biocompatibility characteristics and no significant systemic toxicity in mice, further supporting the potential future use of this material in nanomedicine applications.

\section{Conclusion}

We have demonstrated the preparation of an FA-andICG-covalently conjugated, RSV-encapsulated, integrated theranostic agent for targeting tumor imaging and therapy in vitro and in vivo. FA-RIPNPs exhibited improved photo-, colloidal and heat stabilities compared to free RSV and ICG. FA modification facilitated a highly efficient cellular uptake of FA-RIPNPs via FA RME. Moreover, FA-RIPNPs demonstrated a significantly prolonged circulation time in the bloodstream, which may overcome the generally short circulation times of free RSV and ICG. FA-RIPNPs as fluorescence imaging probes successfully enabled real-time and prolonged nanodrug tumor targeting and accumulation via generation of an in vivo imaging system. Furthermore, in vitro and in vivo studies showed that, compared with free RSV, FA-RIPNPs feature excellent anticancer efficacy induced by apoptosis. Taken in concert, the results obtained indicate that FA-RIPNPs may constitute a promising
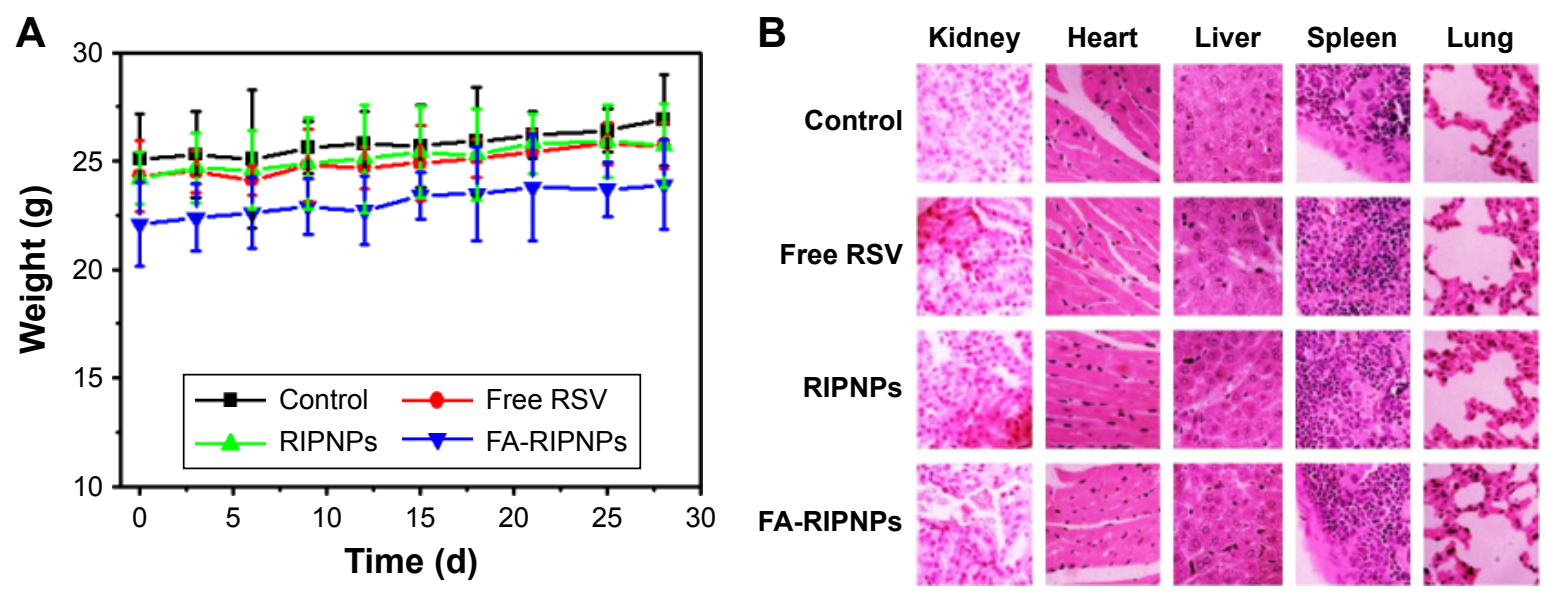

Figure 10 (Continued) 

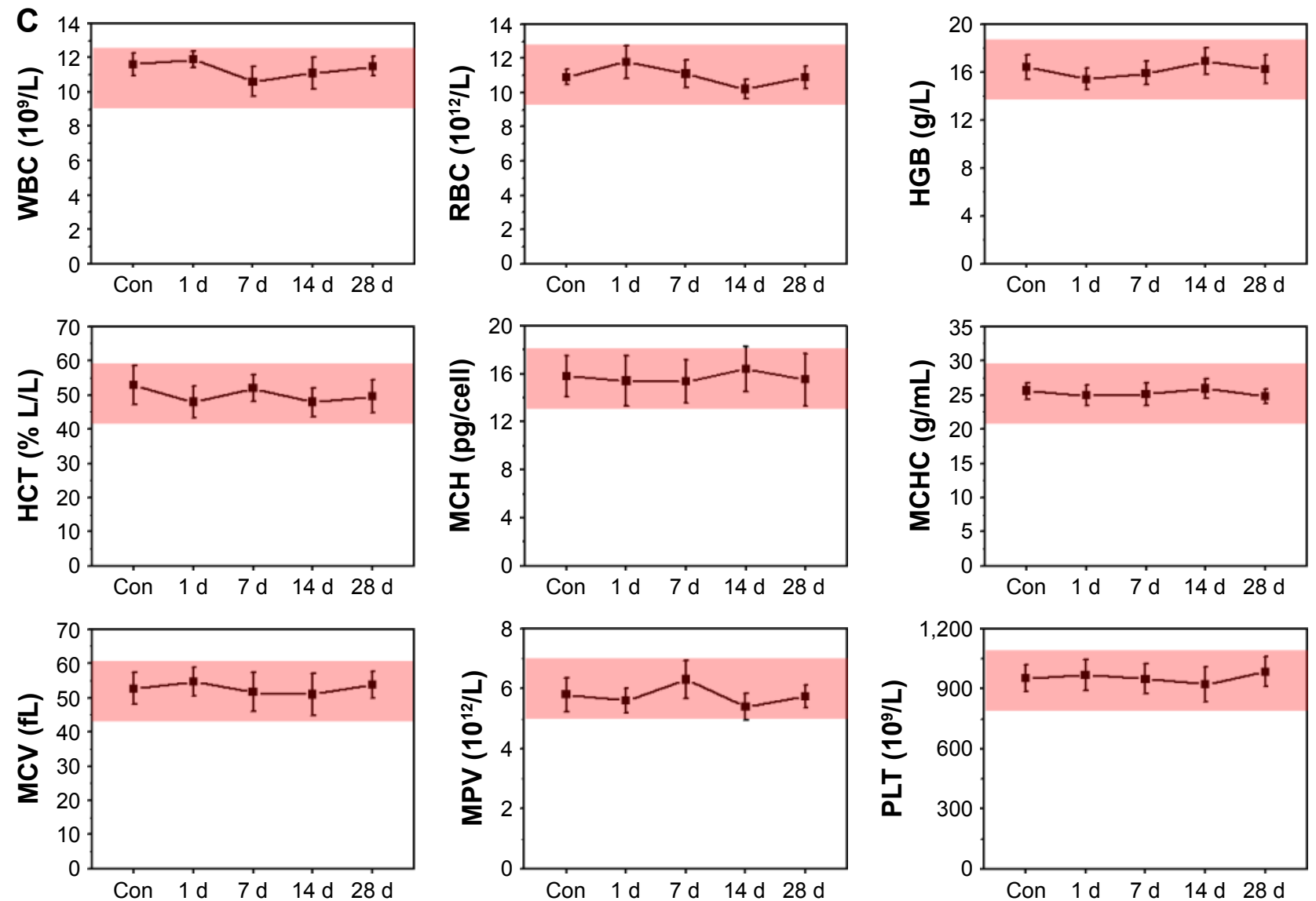

Figure 10 In vivo toxicity.

Notes: (A) Body weights of tumor-bearing mice after tail vein injection with control (saline), free RSV, RIPNPs and FA-RIPNPs. (B) Representative H-E-stained images of major organs, including heart, liver, spleen, lungs and kidneys. (C) Complete blood counts. Blood levels of WBCs, RBCs, HGB, HCT, MCH, MCHC, MCV, MPV and PLT of Con and FA-RIPNP-treated mice were measured $(n=5)$. Red areas indicate the normal reference ranges for healthy female $B A L B / c$ mice.

Abbreviations: Con, control; RSV, resveratrol; FA, folic acid; ICG, indocyanine green; PLGA, poly(D,L-lactide-co-glycolide); NPs, nanoparticles; FA-RIPNPs, FA-RSV/ICGPLGA-lipid NPs; H-E, hematoxylin and eosin; WBC, white blood cells; RBC, red blood cells; HGB, hemoglobin; HCT, hematocrit; MCH, mean corpuscular hemoglobin; $\mathrm{MCHC}$, mean corpuscular hemoglobin concentration; MCV, mean corpuscular volume; MPV, mean platelet volume; PLT, platelets.

integrated theranostic system useful in future nanomedicine applications for tumor therapy.

\section{Acknowledgment}

The authors acknowledge Doctor Yajie Wang from Beijing Tiantan Hospital of Capital Medical University for his kind help in completing blood count detection.

\section{Disclosure}

The authors report no conflicts of interest in this work.

\section{References}

1. Guo J, Gao X, Su L, et al. Aptamer-functionalized PEG-PLGA nanoparticles for enhanced anti-glioma drug delivery. Biomaterials. 2011; 32(31):8010-8020.

2. Lu YJ, Wei KC, Ma CC, Yang SY, Chen JP. Dual targeted delivery of doxorubicin to cancer cells using folate-conjugated magnetic multiwalled carbon nanotubes. Colloids Surf B Biointerfaces. 2012;89(1): $1-9$.
3. Weissleder R, Tung CH, Mahmood U, Bogdanov A Jr. In vivo imaging of tumors with protease-activated near-infrared fluorescent probes. Nat Biotechnol. 1999;17(4):375-378.

4. Hilderbrand SA, Weissleder R. Near-infrared fluorescence: application to in vivo molecular imaging. Curr Opin Chem Biol. 2009;14(1): 71-79.

5. Liu Y, Bauer AQ, Akers WJ, et al. Hands-free, wireless goggles for nearinfrared fluorescence and real-time image-guided surgery. Surgery. 2011; 149(5):689-698.

6. Schaafsma BE, Mieog JS, Hutteman M, et al. The clinical use of indocyanine green as a near - infrared fluorescent contrast agent for imageguided oncologic surgery. J Surg Oncol. 2011;104(3):323-332.

7. Vahrmeijer AL, Hutteman M, van der Vorst JR, van de Velde CJ, Frangioni JV. Image-guided cancer surgery using near-infrared fluorescence. Nat Rev Clin Oncol. 2013;10(9):507-518.

8. Ma Y, Tong S, Bao G, Gao C, Dai Z. Indocyanine green loaded SPIO nanoparticles with phospholipid-PEG coating for dual-modal imaging and photothermal therapy. Biomaterials. 2013;34(31):7706-7714.

9. Nguyen QT, Tsien RY. Fluorescence-guided surgery with live molecular navigation - a new cutting edge. Nat Rev Cancer. 2013;13(9):653-662.

10. Imai T, Takahashi K, Fukura H, Morishita Y. Measurement of cardiac output by pulse dye densitometry using indocyanine green: a comparison with the thermodilution method. Anesthesiology. 1997;87(4): 816-822. 
11. Lau L, Christophi C, Muralidharan V. Intraoperative functional liver remnant assessment with indocyanine green clearance: another toehold for climbing the "ALPPS". Ann Surg. 2015;261(2):e43-e45.

12. Raabe A, Beck J, Gerlach R, Zimmermann M, Seifert V. Near-infrared indocyanine green video angiography: a new method for intraoperative assessment of vascular flow. Neurosurgery. 2003;52(1):132-139.

13. Zheng X, Zhou F, Wu B, Chen WR, Xing D. Enhanced tumor treatment using biofunctional indocyanine green-containing nanostructure by intratumoral or intravenous injection. Mol Pharm. 2012;9(3):514-522.

14. Zheng M, Yue C, Ma Y, et al. Single-step assembly of DOX/ICG loaded lipid-polymer nanoparticles for highly effective chemo-photothermal combination therapy. ACS Nano. 2013;7(3):2056-2067.

15. Hill TK, Abdulahad A, Kelkar SS, et al. Indocyanine green-loaded nanoparticles for image-guided tumor surgery. Bioconjug Chem. 2015; 26(2):294-303.

16. Pang X, Wang J, Tan X, et al. Dual-modal imaging-guided theranostic nanocarriers based on indocyanine green and mTOR inhibitor rapamycin. ACS Appl Mater Interfaces. 2016;8(22):13819-13829.

17. Bahmani B, Lytle CY, Walker AM, Gupta S, Vullev VI, Anvari B. Effects of nanoencapsulation and PEGylation on biodistribution of indocyanine green in healthy mice: quantitative fluorescence imaging and analysis of organs. Int J Nanomedicine. 2013;8(1):1609-1620.

18. Chen J, Liu C, Zeng G, et al. Indocyanine green loaded reduced graphene oxide for in vivo photoacoustic/fluorescence dual-modality tumor imaging. Nanoscale Res Lett. 2016;11(1):1-11.

19. Wang G, Zhang F, Tian R, et al. Nanotubes-embedded indocyanine green-hyaluronic acid nanoparticles for photoacoustic-imaging-guided phototherapy. ACS Appl Mater Interfaces. 2016;8(8):5608-5617.

20. Mundra V, Peng Y, Rana S, Natarajan A, Mahato RI. Micellar formulation of indocyanine green for phototherapy of melanoma. J Control Release. 2015;220(pt A):130-140.

21. Frémont L. Biological effects of resveratrol. Life Sci. 2000;66(8): 663-673.

22. Ji Q, Liu X, Fu X, et al. Resveratrol inhibits invasion and metastasis of colorectal cancer cells via MALAT1 mediated Wnt/ $\beta$-catenin signal pathway. PLoS One. 2013;8(11):e78700.

23. Riccioni G, Gammone MA, Tettamanti G, Bergante S, Pluchinotta FR, D'Orazio N. Resveratrol and anti-atherogenic effects. Int J Food Sci Nutr. 2015;66(6):603-610.

24. Athar M, Back JH, Tang X, et al. Resveratrol: a review of preclinical studies for human cancer prevention. Toxicol Appl Pharmacol. 2007; 224(3):274-283

25. Gomez LS, Zancan P, Marcondes MC, et al. Resveratrol decreases breast cancer cell viability and glucose metabolism by inhibiting 6-phosphofructo-1-kinase. Biochimie. 2013;95(6):1336-1343.

26. Wang G, Dai F, Yu K, et al. Resveratrol inhibits glioma cell growth via targeting oncogenic microRNAs and multiple signaling pathways. Int J Oncol. 2015;46(4):1739-1747.

27. Venuti V, Cannavà $\mathrm{C}$, Cristiano $\mathrm{MC}$, et al. A characterization study of resveratrol/sulfobutyl ether- $\beta$-cyclodextrin inclusion complex and in vitro anticancer activity. Colloids Surf B Biointerfaces. 2014; 115(115C):22-28.

28. Sinha D, Sarkar N, Biswas J, Bishayee A. Resveratrol for breast cancer prevention and therapy: preclinical evidence and molecular mechanisms. Semin Cancer Biol. Epub 2016 Jan 13.

29. Sanna V, Roggio AM, Siliani S, et al. Development of novel cationic chitosan-and anionic alginate-coated poly (D, L-lactide-co-glycolide) nanoparticles for controlled release and light protection of resveratrol. Int J Nanomedicine. 2012;7(2):5501-5516.

30. Chen J, Liu H, Zhao C, et al. One-step reduction and PEGylation of graphene oxide for photothermally controlled drug delivery. Biomaterials. 2014;35(18):4986-4995.

31. Deng R, Yi H, Fan F, et al. Facile exfoliation of $\mathrm{MoS}_{2}$ nanosheets by protein as a photothermal-triggered drug delivery system for synergistic tumor therapy. RSC Adv. 2016;6:77083-77092.
32. Summerlin N, Qu Z, Pujara N, et al. Colloidal mesoporous silica nanoparticles enhance the biological activity of resveratrol. Colloids Surf B Biointerfaces. 2016;144:1-7.

33. Soo E, Thakur S, Qu Z, Jambhrunkar S, Parekh HS, Popat A. Enhancing delivery and cytotoxicity of resveratrol through a dual nanoencapsulation approach. J Colloid Interface Sci. 2016;462:368-374.

34. Summerlin N, Soo E, Thakur S, Qu Z, Jambhrunkar S, Popat A. Resveratrol nanoformulations: challenges and opportunities. Int J Pharm. 2015;479(2):282-290.

35. Fan NC, Cheng FY, Ho JA, Yeh CS. Photocontrolled targeted drug delivery: photocaged biologically active folic acid as a light-responsive tumor-targeting molecule. Angew Chem Int Ed Engl. 2012;51(35): 8806-8810.

36. Bertrand N, Wu J, Xu X, Kamaly N, Farokhzad OC. Cancer nanotechnology: the impact of passive and active targeting in the era of modern cancer biology. Adv Drug Deliv Rev. 2014;66(1):2-25.

37. Zhang Z, Berezin MY, Kao JLF, d'Avignon A, Bai M, Achilefu S. Near - infrared dichromic fluorescent carbocyanine molecules. Angew Chem Int Ed Engl. 2008;47(19):3584-3587.

38. Zhang Z, Kao J, D'Avignon A, Achilefu S. Understanding dichromic fluorescence manifested in certain indocyanine green (ICG) analogs. Pure Appl Chem. 2010;82(1):307-311.

39. Zheng $\mathrm{C}$, Zheng $\mathrm{M}$, Gong $\mathrm{P}$, et al. Indocyanine green-loaded biodegradable tumor targeting nanoprobes for in vitro and in vivo imaging. Biomaterials. 2012;33(22):5603-5609.

40. García-Díaz M, Foged C, Nielsen HM. Improved insulin loading in poly (lactic-co-glycolic) acid (PLGA) nanoparticles upon self-assembly with lipids. Int J Pharm. 2015;482(1):84-91.

41. Simpson CA, Salleng KJ, Cliffel DE, Feldheim DL. In vivo toxicity, biodistribution, and clearance of glutathione-coated gold nanoparticles. Nanomedicine. 2013;9(2):257-263.

42. Cheng L, Gong H, Zhu W, et al. PEGylated Prussian blue nanocubes as a theranostic agent for simultaneous cancer imaging and photothermal therapy. Biomaterials. 2014;35(37):9844-9852.

43. Gillies ER, Fréchet JMJ. pH-responsive copolymer assemblies for controlled release of doxorubicin. Bioconjug Chem. 2005;16(2):361-368.

44. Zhang X, Yang P, Dai Y, et al. Multifunctional Up-converting nanocomposites with smart polymer brushes gated mesopores for cell imaging and thermo/pH dual-Responsive drug controlled release. Adv Funct Mater. 2013;23(33):4067-4078.

45. Dong J, Wang Y, Zhang J, et al. Multiple stimuli-responsive polymeric micelles for controlled release. Soft Matter. 2013;9(2):370-373.

46. Bhat KPL, Kosmeder JW, Pezzuto JM. Biological effects of resveratrol. Antioxid Redox Signal. 2001;3(6):1041-1064.

47. Ding H, Ma Y. Role of physicochemical properties of coating ligands in receptor-mediated endocytosis of nanoparticles. Biomaterials. 2012; 33(23):5798-5802.

48. Zheng M, Zhao P, Luo Z, et al. Robust ICG theranostic nanoparticles for folate targeted cancer imaging and highly effective photothermal therapy. ACS Appl Mater Interfaces. 2014;6(9):6709-6716.

49. Gu H, Wang X, Rao S, et al. Gambogic acid mediates apoptosis as a $\mathrm{p} 53$ inducer through down-regulation of mdm2 in wild-type p53expressing cancer cells. Mol Cancer Ther. 2008;7(10):3298-3305.

50. Sasidharan A, Panchakarla LS, Chandran P, et al. Differential nanobio interactions and toxicity effects of pristine versus functionalized graphene. Nanoscale. 2011;3(6):2461-2464.

51. Xu C, Ding Y, Ni J, Yin L, Zhou J, Yao J. Tumor-targeted docetaxel-loaded hyaluronic acid-quercetin polymeric micelles with p-gp inhibitory property for hepatic cancer therapy. RSC Adv. 2016;6(33):27542-27556.

52. Lin G, Wang X, Yin F, Yong KT. Passive tumor targeting and imaging by using mercaptosuccinic acid-coated near-infrared quantum dots. Int $J$ Nanomedicine. 2015;10:335-345. 
International Journal of Nanomedicine

Dovepress

\section{Publish your work in this journal}

The International Journal of Nanomedicine is an international, peerreviewed journal focusing on the application of nanotechnology in diagnostics, therapeutics, and drug delivery systems throughout the biomedical field. This journal is indexed on PubMed Central, MedLine, CAS, SciSearch ${ }^{\circledR}$, Current Contents ${ }^{\circledR} /$ Clinical Medicine,
Journal Citation Reports/Science Edition, EMBase, Scopus and the Elsevier Bibliographic databases. The manuscript management system is completely online and includes a very quick and fair peer-review system, which is all easy to use. Visit http://www.dovepress.com/ testimonials.php to read real quotes from published authors.

Submit your manuscript here: http://www.dovepress.com/international-journal-of-nanomedicine-journal 